\title{
PREVALÊNCIA DE SINTOMAS DEPRESSIVOS NA PERIMENOPAUSA
}

\section{DEPRESSIVE SYMPTOMS PREVALENCE IN THE PERIMENOPAUSE}

\author{
Genilda Garcia Calvoso* \\ Roberto Calvoso Júnior** \\ Alberto Olavo Advíncula Reis*** \\ José Mendes Aldrighi****
}

Calvoso GG, et al. Prevalência de Sintomas Depressivos na Perimenopausa. Rev Bras

Crescimento Desenvolvimento Hum. 2008; 18(3): 339-345

\section{RESUMO:}

Objetivo: realizar revisão sistemática de estudos de prevalência dos sintomas depressivos na perimenopausa. Método: A pesquisa foi efetuada a partir das bases de dados MEDLINE e EMBASE. Foram incluídos estudos transversais originais com populações adultas (idade de 40 a 55 anos), no período de 1996 a 2006, que estimaram a prevalência de sintomas depressivos na perimenopausa nos idiomas: inglês, francês, espanhol e português. A seleção dos estudos pela estratégia de busca foi feita de forma independente por dois avaliadores. Foram incluídos os estudos que preencheram os seguintes critérios metodológicos: características epidemiológicas, definições de perimenopausa, instrumentos válidos utilizados para sintomas depressivos. Resultados: Após a avaliação de 1.345 artigos, restaram apenas cinco que caracterizaram uma população de 11.020 mulheres com idades entre 40 e 60 anos. Constatamos que a prevalência de sintomas depressivos na perimenopausa variou entre 19\% e 73\%. Conclusão: A presente revisão sistemática mostrou expressiva variação na prevalência de sintomas depressivos na perimenopausa; ademais, não foi possível caracterizar se a sintomatologia depressiva na perimenopausa decorreu exclusivamente de flutuações hormonais observadas neste estágio da vida ou de antecedentes prévios de depressão.

Palavras-chave: Menopausa; transição menopausal; perimenopausa; sintomas depressivos; prevalência; revisão sistemática da literatura.

Dissertação apresentada ao Programa de Pós-Graduação em Saúde Pública para obtenção do título de Mestre em Saúde Pública. Área de concentração: Saúde Materno-Infantil - 2007

* $\quad$ Mestre em Saúde Pública. Faculdade de Saúde Pública da Universidade de São Paulo.

** Doutor em Medicina. Coordenador do Curso de Especialização em Saúde da Mulher no Climatério. Departamento de Saúde Materno-Infantil. Faculdade de Saúde Pública da Universidade de São Paulo.

*** Professor Doutor. Departamento de Saúde Materno-Infantil. Faculdade de Saúde Pública da Universidade de São Paulo.

**** Professor Associado. Departamento de Saúde Materno-Infantil. Faculdade de Saúde Pública da Universidade de São Paulo. Correspondência para Genilda Garcia Calvoso, Rua do Símbolo, 16 apto. 151 - Morumbi, São Paulo, SP CEP 05713-570, e-mail: gcalvoso@gmail.com 


\section{ABSTRACT:}

Objective: a systematic review of studies that investigated the prevalence of depressive symptoms in perimenopausal women. Method: The research was conducted in MEDLINE and EMBASE databases. Original transversal studies in from 1996 to 2006 with adult populations were enclosed (age between 40 and 55 years old) and demonstrated prevalence of depressive symptoms in the perimenopause in the following languages: English, French and Portuguese. The studies selection was conducted by two independent evaluators. The inclusion criteria were studies which fulfilled the following methodological criteria: epidemiological characteristics, perimenopause definitions and valid instruments to evaluate depressive symptoms. Results: After the evaluation of 1.345 articles, 1099 were excluded since they did not satisfy the criteria of inclusion, remaining only five articles with a population of 11.020 women with ages between 40 and 60 years and, therefore, part of the studied sample enclosed in perimenopause. This study revealed a prevalence of depressive symptoms in perimenopause, which varied between $19 \%$ and $73 \%$. Conclusion: The present systematic review showed substantial variation in the prevalence of depressive symptoms in perimenopause; in addition, it was not possible to characterize if the depressive symptoms in perimenopause were exclusively due to hormonal fluctuations in this stage life or if related to previous preceding moments of depression.

Key words: Menopause; menopausal transition; perimenopause; depressive symptoms; prevalence; literature systematic review.

\section{INTRODUÇÃO}

A transição menopausal é um estágio do envelhecimento ovariano que se inicia em torno dos 37 anos e termina com a última menstruação da vida da mulher (menopausa), que ocorre, em geral, aos 48 anos. ${ }^{1}$ A perimenopausa, por sua vez, é uma fase situada dentro da transição menopausal, com início aproximado aos 45 anos e término após um ano da menopausa.

Na perimenopausa ocorrem significativas oscilações dos hormônios ovarianos que se responsabilizam por numerosas repercussões sobre o organismo feminino. ${ }^{1-5}$ Entretanto, algumas mulheres mostram-se mais vulneráveis ao surgimento dos sintomas depressi$\operatorname{vos}^{6-19}$ na perimenopausa, permitindo supor que outros fatores poderiam estar envolvidos na sua gênese.
Contrariando a participação do fator endócrino (flutuação hormonal), demonstra-se ainda que o primeiro episódio de depressão na perimenopausa independe das concentrações séricas de hormônios ${ }^{20}$; ademais, outros estudos mostraram que fatores psicológicos exclusivos são comumente relacionados à sintomatologia depressiva, ressaltando entre outros, a ausência de estímulo para metas existenciais. ${ }^{5,21-23}$

Esta revisão sistemática foi motivada por inúmeras inquietudes referentes aos sintomas depressivos na transição e/ou perimenopausa, destacando a prevalência, os fatores envolvidos na sua gênese, especialmente as flutuações hormonais características da perimenopausa, os fatores psicossociais e a reincidência de estados depressivos. 


\section{MÉTODO}

Para realizar a revisão sistemática, definimos a busca por estudos de prevalência ${ }^{24}$, no período de janeiro de 1996 a dezembro de 2006. A busca foi realizada na Biblioteca Cochrane e a Base de Dados de Revisões Sistemáticas ${ }^{25}$, além da $M E D L I N E^{26}$ e EMBASE. ${ }^{27}$

A estratégia para a procura baseou-se nos descritores: menopause, menopausal, premenopause, perimenopause, perimeno-pausal, depressions, depressive, depression, prevalence. $^{28,29}$

A seleção dos estudos foi feita de forma independente por dois avaliadores (médico e psicóloga) com acesso ao nome do autor, instituição e revista ou jornal que publicou o estudo. Inicialmente procedeu-se à análise dos títulos dos artigos e avaliação dos resumos, quando disponíveis.

A partir de então, cada artigo considerado original e/ou elegível foi analisado completamente, de modo a caracterizá-lo quanto aos critérios de inclusão dos estudos até a obtenção de um consenso final entre os avaliadores, a fim de garantir a qualidade metodológica.

Foram definidos como critérios de inclusão: estudos que investigaram mulheres na transição menopausal, com idade média entre 40 e 55 anos $^{30}$, estudos transversais, com mensuração da prevalência de sintomas depressivos na perimenopausa, exclusivamente nos idiomas: inglês, francês, espanhol e português. Foram considerados citações da mesma entidade nosológica os termos transição menopausal $^{30}$ (precoce e tardia), ciclos menstruais irregulares e perimenopausa. O mesmo critério foi utilizado para os termos depressão e sintomas depressivos. ${ }^{31,32}$

Excluíram-se todos os estudos que relacionassem sintomas depressivos secundários a doenças orgânicas e a procedimentos cirúrgicos, pesquisas de intervenção em mulheres sob utilização de fármacos, comparação entre prémenopausa e pós-menopausa sem contemplar perimenopausa, relatos de caso, cartas ao editor, publicações em congressos e artigos nos quais a amostra avaliada contivesse pacientes psiquiátricos, estudos longitudinais e revisões de especialistas.

\section{RESULTADOS}

A estratégia de busca detectou 1.345 estudos, sendo que 1.325 foram identificados por meio de busca eletrônica e 20 por procura manual. Os resultados foram agrupados conforme as seguintes características: número de estudos, participantes, qualidade metodológica, mensuração de sintomas depressivos e prevalência na perimenopausa. Após análise dos dois avaliadores, foram descartados 1.099 estudos, por não serem condizentes com os critérios de inclusão dessa revisão. Restaram 246 estudos elegíveis, que após leitura ainda mais apurada pela qualidade metodológica, resultou na inclusão de apenas cinco estudos na presente revisão sistemática.

Os cinco estudos identificados descreveram critérios para perimenopausa. Entretanto, em nenhum deles foi reportado o tempo de perimenopausa. Todos avaliaram os sintomas depressivos por instrumentos validados. Os estudos mostraram um total de 11.020 participantes, com idades entre 40 e 60 anos, de várias nacionalidades e áreas geográficas definidas pelos seguintes países: Escócia ${ }^{33}$, Tailândia $^{34}$, Chile ${ }^{35}$, Brasil ${ }^{36}$, Japão e Austrá$\mathrm{lia}^{37}$. Relataram dados da prevalência de sintomas depressivos na perimenopausa, que variou entre 19\% na Escócia, 39,2\% no Chile, 47,9\% no Japão, 55\% na Austrália, 67,6\% no Brasil e 73\% na Tailândia.

\section{DISCUSSÃO}

A revisão sistemática assume o papel de um poderoso instrumento para os profissionais 
da saúde poderem adquirir rapidamente as necessárias informações para sua imprescindível atualização. ${ }^{38-40}$

Na presente revisão sistemática a análise da prevalência de sintomas depressivos na perimenopausa constatou 1345 artigos publicados nos últimos dez anos, sendo que após exaustiva leitura apenas cinco estudos versavam sobre a caracterização da prevalência dos sintomas depressivos na perimenopausa: PORTER et al. (1996) ${ }^{33}$, PUNYAHOTRA et al. (1997) $)^{34}$, LAGOS et al. $(1998)^{35}$, PEDRO et al. $(2003)^{36}$, ANDERSON et al (2004). ${ }^{37}$

Foram constatadas populações distintas geograficamente e diversidades nas taxas de prevalência de sintomas depressivos na perimenopausa; na Escócia ${ }^{33}$ a mais baixa, $19 \%$, quando comparada com as do Japão e Austrália $^{37}$, respectivamente 47,9\% e 55\%, tanto na população rural quanto na metropolitana. Entre as latinas, no Chile ${ }^{35}$ 39,2\% e no Brasil $^{36} 67,6 \%$. Nos países asiáticos, o Japão ${ }^{38}$ 47,9\% e a Tailândia ${ }^{34}$ atingiu $73 \%$.

Como limitação destes estudos, deve se destacar que as amostras das pesquisas brasileira $^{34}$, chilena ${ }^{33}$ e tailandesa ${ }^{32}$ envolveram um número reduzido de mulheres, portanto, insuficiente para permitir generalizações quanto à alta prevalência observada.

Quanto aos instrumentos empregados nos estudos para identificar os sintomas depressivos ressalta-se que todos eram valida$\operatorname{dos}^{31-35,39-43}$ e foram também utilizadas escalas de auto-avaliação. Estes instrumentos são mais adequados para detectar formas leves de depressão ${ }^{45}$, além de serem auto-aplicáveis, o que enaltece a circunstância da percepção sentida pela pessoa naquele momento. Destaca-se ainda as desvantagens como fidedignidade, cooperação, certo grau educacional e ausência de psicopatologia grave. ${ }^{44}$

Nessa revisão, dois estudos utilizaram a escala de rastreamento de depressão ${ }^{31,33,39}$, cuja principal finalidade é a de aferir a prevalência atual de sintomas depressivos ("point prevalence”). Outro estudo utilizou a lista de sintomas adaptados de Kaufert e Syrotuik ${ }^{32,41}$, contudo, a utilização de listas, deve ser muita cautelosa, principalmente diante de situações como o analfabetismo, problemas de linguagem e diferenças culturais. ${ }^{46}$

Em contrapartida, nos últimos 30 anos vários instrumentos padronizados de avaliação diagnóstica foram propostos e vêm sendo utilizados em pesquisa clínica. Como as mudanças observadas nas mulheres na transição menopausal podem ser tanto físicas como psíquicas, os instrumentos que avaliam itens somáticos podem se tornar variáveis de confusão ${ }^{44}$ para sintomas depressivos. Os resultados dessa revisão mostraram que dois estudos ${ }^{34,35}$ utilizaram esse tipo de instrumento. ${ }^{40,41,43}$

De maneira geral, esta padronização permite que diferentes profissionais em diferentes serviços e/ou países possam utilizar os mesmos instrumentos, fazendo com que os resultados obtidos sejam homogêneos e, portanto de fácil comparação. ${ }^{47}$ Contudo, existe uma variedade de fontes de erro que podem resultar em baixa confiabilidade, destacandose a diferença fundamental entre as práticas diagnósticas para pesquisadores e clínicos.

Uma importante limitação observada nesta revisão é que os estudos não contemplaram em seus métodos a avaliação dos antecedentes psicológicos das mulheres. Nenhum deles fez referência aos antecedentes de tensão pré-menstrual, ocorrência de transtornos disfóricos prémenstruais e depressão pós-parto. Assim, esta temática carece de investigações futuras.

Assim, esta revisão sistemática evidenciou expressiva variação na prevalência de sintomas depressivos na perimenopausa, atingindo taxas entre $19 \%$ e $73 \%$. Ademais, não se pode caracterizar se a sintomatologia depressiva na perimenopausa decorre exclusivamente de flutuações hormonais observadas neste estágio da vida ou de antecedentes prévios de depressão ou de ambos os fatores. 


\section{REFERÊNCIAS}

1. Aldrighi JM, Lima SMRR, Fráguas JrR. Menopausa e climatério. In: Fráguas JrR., Figueiró JAB e cols. Depressões em medicina interna e em outras condições médicas: depressões secundárias. São Paulo: ed. Atheneu, 2000; 363-68.

2. Soules MR, Sherman S, Parrott E, Rebar R, Santoro N, Utian W, Woods N. Stages of Reproductive Aging Workshop (STRAW). J Womens Health Gend Based Med. 2001; Nov;10(9):843-8.

3. Dennerstein L, Lehert P, Guthrie J. The effects of the menopausal transition and biopsychosocial factors on well-being. Arch Women Ment Health 2002; 5:1522.

4. Calvoso JrR, Aldrighi JM, Negrão CE, Trombetta IC, Ramires JAF. Efeito do estradiol sobre as respostas cardiopulmonar e metabólica em mulheres normotensas após a menopausa submetidas à cicloergoespirometria. Arquivos Brasileiros de Cardiologia v. 84, n. 1, p. 15-19, 2005.

5. Favarato MEC, Hueb WA, Aldrighi JM. Qualidade de vida em portadores de doença arterial coronária submetidos a diferentes tratamentos. Rev. Assoc. Med. Bras., July/Aug. 2006; vol.52, no.4, p.236-241.

6. Kessler RC, Mc McGonagle K, Zhao S, Nelson CB, Hughes M, Eshleman S, et al. Lifetime and 12-month prevalence of DSM III-R psychiatric disorders in the United States. Arch Gen Psychiatry 1994;51:8-19.

7. Harlow BL, Cohen LS, Otto MW, Spiegelman D, Cramer DW. Prevalence and predictors of depressive symptoms in older premenopausal women. Arch Gen Psychiatry 1999;56:418-24.

8. Soares CN, Almeida OP, Joffe H, Cohen LS. Efficacy of estradiol for the treatment of depressive disorders in perimenopausal women: a double-blind, randomized, placebo-controlled trial. Arch Gen Psychiatry 2001;58:529-34.

9. Born L, Steiner M. The relationship between menarche and depression in adolescence. CNS Spectrums 2001;6:126-38.

10. Cohen LS, Soares CN, Joffe H. Diagnosis and management of mood disorders during the menopausal transition. In: The American Journal of Medicine. Vol. 118, n. 12, supl. 2, p. 9397, 2005.

11. Freeman EW, Sammel MD, Rinaudo PJ, Sheng L. Premenstrual syndrome as a predictor of menopausal symptoms. PMS and Menopausal Symptoms. Vol.103, n.5, part 1, may 2004.

12. Richards M, Rubinow DR, Daly RC, Schmidt, PJ. Premenstrual and perimenopausal depression. Am J Psychiatry. 2006; 163:133-137.

13. Avis NE, Brambilla D, McKinlay SM, Vass K. A longitudinal analysis of the association between menopause and depression: results from the Massachusetts Women's Health Study. Arch Epidemiol 1994; 4:214-220.

14. Schmidt PJ, Haq N, Rubinow DR. A longitudinal evaluation of the relationship between reproductive status and mood in perimenopausal women. Am J Psychiatry. 161:12, Dec 2004.

15. Bromberger JT, Assmann SF, Avis NE, Schocken M, Kravitz HM, Cordal A. Persistent mood symptoms in a multiethnic community cohort of preand perimenopausal women. Am J Epidemiol. 2003 Aug 15;158(4): 347-56.

16. Suau GM, Normandia R, Rodriguez R, Romaguera J, Segarra L. Depressive symptoms and risk factors among 
perimenopausal women. P R Health Sci J. 2005 Sep;24(3):207-10.

17. Amore M, Di Donato P, Papalini A, Berti A, Palareti A, Ferrari G, Chirico C, De Aloysio D. Psychological status at the menopausal transition: an Italian epidemiological study. Maturitas. 2004; Jun 15;48(2):115-24.

18. Dennerstein L. Well-being, symptoms and the menopausal transition. Maturitas. 1996; Mar;23(2): 147-57.

19. Cohen LS, Soares CN, Allison F, Vitonis AF, Otto MW, Harlow BL. Risk for new onset of depression during the menopausal transition. Arch Gen Psychiatry. 2006; 63:385-390.

20. Schmidt PJ, Murphy JH, Danaceau MA, Simpson SC. Basal plasma hormone levels in depressed perimenopausal women. Psychoneuroendocrinology. 2002; 27:907-920.

21. Evangelista EI, Cezarino PYA, Mieli MPA, Aldrighi JM. Autonomia e climatério. Femina vol 33 n. 1. Jan 2005; 67-68.

22. Pan HA, Wu MH, Hsu CC, Yao BL, Huang KE. The perception of menopause among women in Taiwan. Maturitas. 2002 Apr 25;41(4):269-74.

23. Rasgow N. Is there an increased risk of depression during the menopause transition? In: Menopause: The Journal of the NAMS. Vol.3, n. 1, p. 1-3, 2007.

24. Pereira MG. Morbidade. In: Pereira MG. Epidemiologia teoria e prática. 8 ed. 2005:76-104.

25. Biblioteca Cochrane. Disponível em URL: http://cochrane.bireme.br/index.php. Acessado em 20 de janeiro de 2007.

26. MEDLINE - Medical Literature Analysis and Retrieval System Online. Disponível em URL: National Library of Medicine (NLM).

27. EMBASE - Excerta Médica Database. Disponível em URL: http:// www1.elsevier.com/homepage/sah/spd/ site/locate_embase.html

28. DeCS - Descritores em Ciências da Saúde. Disponível em URL: http:// decs.bvs.br/. Acessado em 4 de fevereiro de 2005.

29. Alphabetical list of titles of Cochrane Reviews. Disponível em URL: http:// www.centrocochranedobrasil.org. Acessado em 17 de maio de 2005.

30. Gracia CR, Sammel MD, Freeman EW, Lin H, Langan E, Kapoor S, Nelson DB. Defining menopause status: creation of a new definition to identify the early changes of the menopausal transition. Menopause 2005; 12(2):128-35.

31. Porter M, Penney GC, Russell D, Russeli E. a population based survey of women's experience of the menopause. British Journal of Obstetrics and Gynaecology Oct 1996 (103): 1025-1028.

32. Punyahotra S, Dennerstein L, Lehert P. Menopausal experiences of Thai women. Part 1: Symptoms and their correlates. Maturitas 1997; 26: 1-7.

33. Lagos PX, Navarro HN, Illanes VE, Bustos ML. Prevalencia de síntomas biológicos y psicológicos en mujeres perimenopáusicas, según estrato socioeconómico, en la ciudad de Temuco. Rev Méd Chile 1998; 126: 1189-1194.

34. Pedro AO, Pinto-Neto AM, Costa-Paiva LHS, Osis MJD, Hardy EE. Climacteric syndrome: a population-based study in Brazil. Rev Saúde Pública Dez. 2003; v.37 (6):735-42.

35. Anderson D, Yoshizawa T, Golischewski S, Atogami F, Courtney M. Relationship between menopausal symptoms and menopausal status in Australian and Japanese women:preliminary analysis. Nursing and Health sciences 2004; 6, 173-180. 
36. Hearst N, Grady D, Barron HV, Kerlikowske K. Pesquisas com dados existentes: análise de dados secundários, estudos suplementares e revisões sistemáticas. In: Hulley SB et al. Delineando a pesquisa clínica: uma abordagem epidemiológica. 2.ed. Porto Alegre: Artmed, 2003; 225-244.

37. Galvão CM, Sawada NO, Trevizan MA. Revisão sistemática: recurso que proporciona a incorporação das evidências na prática da enfermagem. Rev.Latino-Am. Enfermagem vol.12 n3 Ribeirão Preto May/June 2004.

38. Martins HS, Olmos RD. Metanálise princípios, vantagens e limitações. In: Benseñor IM \& Lotufo PA.

Epidemiologia: abordagem prática. São Paulo: ed. Savier, 2005; 245-272.

39. Radloff LS. The CES-D Scale: a selfreport depression scale for research in the general population. Appl Psychol Meas 1977; 1(3):385-401.

40. Greene JG Constructing a standard climacteric scale. Maturitas 29 (1998) 25-31.

41. Kaufert P, Syrotuik J. Symptom reporting at the menopause. Soc Sci Med 1981;15:173-84.

42. Boulet MJ, Oddens BJ, Lehert P, Vemer HM, Visser A. Climacteric and menopause in seven South-east Asian countries. Maturitas 1994;19:157-76.
43. Utian WH, Schiff I. North American Menopause Society - Gallup survey on women's knowledge, information sources, and attitudes to menopause and hormone replacement therapy. Menopause 1994;1:39-48.

44. Calil HM, Pires MLN. Aspectos gerais das escalas de avaliação de depressão. Rev Psiquiatr Clin 1998; 25:240-4.

45. Rabkin JG, Klein DF. The Clinical Measurement of Depressive Disorders. In: The Measurement of Depression. The Guilford Press. New York, 30-83, 1987.

46. Blay SL. Escalas de avaliação utilizadas em múltiplos quadros. Rev Psiq Clin 1999; Mar/Abr Vol 26 (2). Disponível em URL: http://www.hcnet.usp.br/ipq/ revista/r262/artigo(106).htm. Acessado em 17 de janeiro de 2007.

47. Jorge MR, Custódio O. Utilidade das escalas de avaliação para clínicos e pesquisadores. Rev Psiq Clin 1999; Mar/ Abr Vol 26 (2). Disponível em URL: http://www.hcnet.usp.br/ipq/revista/ r262/artigo(106).htm. Acessado em 17 de janeiro de 2007.

48. Silveira DX, Jorge MR. Propriedades psicométricas da escala de rastreamento populacional para depressão CES-D em populações clínica e não-clínica de adolescentes e adultos jovens. Rev. Psiq. Clin. 25 (5) Set/Out 1998. Disponível em URL: http://www.hcnet.usp.br/ipq/ revista/r255/depre255c.htm. Acessado em 17 de janeiro de 2007.

Recebido em: 08/09/2008 Modificado em: 06/12/2008 Aceito em: 19/12/2008 